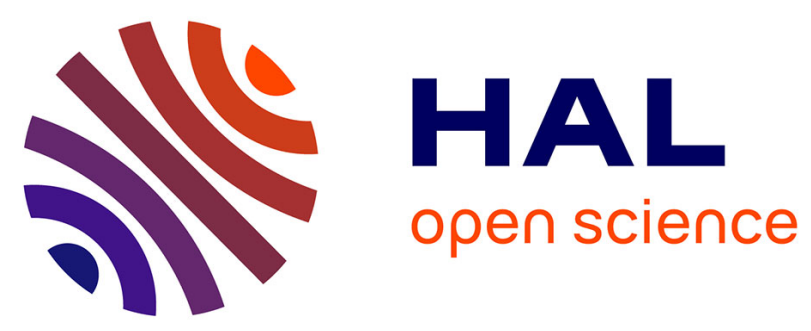

\title{
Electromagnetic Time Reversal as a Correlation Estimator: Improved Metrics and Design Criteria for Fault Location in Power Grids

\author{
Shaoyin He, Andréa Cozza, Yanzhao Xie
}

\section{- To cite this version:}

Shaoyin He, Andréa Cozza, Yanzhao Xie. Electromagnetic Time Reversal as a Correlation Estimator: Improved Metrics and Design Criteria for Fault Location in Power Grids. IEEE Transactions on Electromagnetic Compatibility, 2019, 62 (2), pp.598 - 611. 10.1109/TEMC.2019.2904841 . hal02051766

HAL Id: hal-02051766

https://hal-centralesupelec.archives-ouvertes.fr/hal-02051766

Submitted on 28 Feb 2019

HAL is a multi-disciplinary open access archive for the deposit and dissemination of scientific research documents, whether they are published or not. The documents may come from teaching and research institutions in France or abroad, or from public or private research centers.
L'archive ouverte pluridisciplinaire HAL, est destinée au dépôt et à la diffusion de documents scientifiques de niveau recherche, publiés ou non, émanant des établissements d'enseignement et de recherche français ou étrangers, des laboratoires publics ou privés. 


\title{
Electromagnetic Time Reversal as a Correlation Estimator: Improved Metrics and Design Criteria for Fault Location in Power Grids
}

\author{
Shaoyin He, Andrea Cozza, Senior Member, IEEE, Yanzhao Xie, Member, IEEE
}

\begin{abstract}
Recently published papers have proven the effectiveness of Electromagnetic time reversal (EMTR) in locating the position of faults such as phase-to-ground shunt connections in power grids. EMTR directly transposes the idea of focusing energy back to its source introduced in original time-reversal methods. The current interpretation of EMTR, based on metrics measuring energy or peak-amplitude of focusing, is shown to suffer from ambiguities that increase the risk of inaccurate fault location. After pointing out under what conditions time-reversal focusing occurs, an original frequency-domain reformulation of EMTR is introduced, showing that EMTR should more accurately be interpreted as a correlation estimator. New metrics based on this observation are introduced, taking into account the inhomogeneous transmission of electrical energy throughout complex networks, enabling a direct quantitative evaluation of the likelihood of locating a fault. Extensive numerical simulations confirm that the proposed formulation systematically improves the reliability of EMTR location estimates when faults occur in power grids of realistic complexity, highlighting the accrued risk that comes with the use of metrics that measure the scale of time-reversal focusing rather than its quality.
\end{abstract}

Index Terms-Fault location, power grids, time reversal.

\section{INTRODUCTION}

$\mathbf{T}$ HE proper operation of power grids can be severely disrupted by events such as shunt faults where two or more conductors, subject to different potentials, become electrically connected through a low-impedance path, e.g., because of electrical arcs or phase-to-ground faults.

The occurrence of such shunt faults is followed by transient signals that can be detected by a monitoring station, or probe, and subsequently used in order to estimate the fault position. A large number of methods has been introduced in the last decades for fault location in power grids, among which those based on travelling waves are of interested in the context of this paper [1]-[4].

A recent proposal for fault location is Electromagnetic Time Reversal (EMTR) [5], which is inspired by the idea of timereversal (TR) focusing [6], [7]. Its main appeal is its ability to locate faults from the transient signals they generate, as measured from a single probe. Its performance has been shown to depend on what kind of metrics are used, e.g., by monitoring

S. He and Y. Xie are with Xi' an Jiaotong University, School of Electrical Engineering, West Xianning Road No.28, Xi'an, Shaanxi Province, 710049, China

A. Cozza is with the Group of Electrical Engineering - Paris (GeePs), CentraleSupelec, 3 rue Joliot-Curie, Plateau de Moulon, 91192 Gif-sur-Yvette CEDEX, France

Contact e-mails: yzxie@xjtu.edu.cn, andrea.cozza@ieee.org maximum focused energy or peak amplitude [8]. While EMTR has been presented as an effective fault-location method [9][11], none of the results available in the literature has so far provided clear design criteria and necessary conditions to ensure accurate fault location.

This paper starts by revisiting in Sec. II the definitions employed in EMTR, pointing out some fundamental discrepancies with TR, which are shown to lead to inaccurate expectations, in particular in the ability of EMTR to provide a single focusing peak, and therefore a single candidate position for the fault. Sec. III presents an alternative interpretation of EMTR, showing how it basically mimics the properties of correlation functions, which are meant to only identify similarity. The fact that currently used EMTR norms do not take into account the existence of scale terms related to the inhomogeneous propagation of transient energy throughout a distribution network, is shown to potentially lead to spurious fault locations. Moreover, the unacknowledge effect of strongly frequency-dependent fault transient signals is also argued to dramatically reduced EMTR location performance, while requiring access to unnecessarily wideband data.

Based on these observations, Sec. IV introduces two alternative norms that are subsequently shown to systematically outperform those of energy- and peak amplitude-based norms. The necessary conditions for their accurate use are discussed, showing that these metrics can be promptly adopted without introducing any further complexity in EMTR location processing. Moreover, they lend themselves to quantitative interpretation, since they represent correlation functions. On the contrary, current EMTR norms require an a posteriori normalization, before being able to locate a fault. As new candidate fault positions are tested, the result of this normalization process is bound to change, and is therefore qualitative in nature. Moreover, this kind of normalization does not warrant a direct quantitative interpretation, since it does not correspond to a well-define quantity, such as a correlation function. In turn, this risks making it difficult to introduce a quantitative criterion to decide whether a fault has been reliably located or not.

Extensive simulations results are analyzed in Sec. V, for three grids of increasing complexity, confirming all the implications and predictions discussed in the previous sections. A remarkable result is the feasibility of accurately locating a fault using only low-frequency information, as low as $30 \mathrm{kHz}$, as opposed to $1 \mathrm{MHz}$ required by previous EMTR norms, which is of practical importance when dealing with real-life lossy 


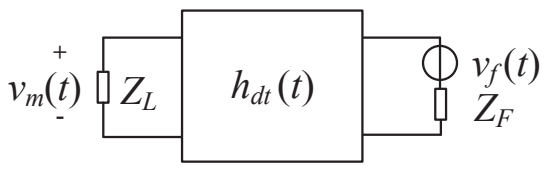

(a)

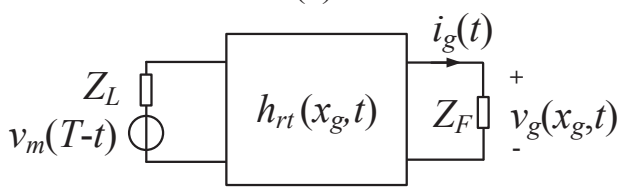

(b)

Fig. 1. Principle of implementation of EMTR to locate faults, and related quantities for its analysis: (a) direct-time phase, during which the fault transient signal is recorded and (b) reversed-time phase, enabling the rate of TR focusing in numerically tested candidate fault positions.

lines.

Finally, Sec. VI argues about the necessary conditions required for EMTR to provide accurate locations, as based on known results for the TR property of self averaging, upon which TR methods rely to control the propagation of signals through complex media. Design guidelines are then discussed in order to allow a better understanding of EMTR limitations.

\section{A BRIEF SUMMARY OF EMTR}

EMTR is based on two phases, schematically represented in Fig. 1, where $Z_{L}$ is the equivalent impedance of the termination load of the transmission lines, usually representing transformers. These were argued in [5], [12] to typically behave as capacitances in the hundreds of $\mathrm{pF}$, in the range of frequencies covered by transient signals. For this reason, $Z_{L}$ will be assumed to be larger than the characteristic impedance $Z_{c}$ of transmission lines by several orders of magnitude. The fault is represented by its Thevenin equivalent circuit, with an impedance $Z_{F}<Z_{c}$, and a voltage generator $v_{f}(t)$ modeling the fault transient.

Transient signals generated by the fault are first recorded, e.g., by monitoring the voltage $v_{m}(t)$ by a probe at one of the terminations of the distribution network, as in Fig. 1(a). The network under test (NUT) is modeled by its impulse response $h_{\mathrm{dt}}(t)$, for direct time. Subsequently, a time-reversed version of the probe-recorded transient signal, e.g., $v_{m}(T-t)$, is injected in numerical models of the NUT, where different candidate fault positions $x_{g}$ are tested; the delay $T$, required to ensure causality, will be dropped hereafter for the sake of simplicity, without loss of generality.

As discussed in [5], EMTR estimates the actual fault position by monitoring the signal energy at each tested fault position $x_{g}$, e.g., by measuring the voltage $v_{g}(t)$, or the current $i_{g}(t)$. During this second (numerical) phase, each system response is modeled by an impulse response $h_{\mathrm{rt}}\left(t ; x_{g}\right)$. The best estimate $\hat{x}_{f}$ is identified as the one that maximizes the energy, i.e.,

$$
\hat{x}_{f}=\underset{x_{g}}{\operatorname{argmax}}\left\|v_{g}\left(t ; x_{g}\right)\right\|
$$

where $\left\|v_{g}(t)\right\|$ was originally the energy of $v_{g}(t)$, eventually evaluated only over a finite time interval [9]. More recent work [8] showed that other norms perform better, by monitoring, e.g., the peak-amplitude of voltage or current at the test fault locations. In the rest of the paper, the energy norm will be referred to as $L_{2}^{2}$, i.e., the $L_{2}$ norm squared, and the peak amplitude as $L_{\infty}$, for the sake of simplicity.

While previous EMTR works have maintained the interpretation introduced by standard TR for the need to actually inject TR signals into the system, an alternative approach will be used throughout this paper, which significantly simplifies this operation. The voltage $v_{g}(t)$ observed across the fault during the second phase can be written as

$$
v_{g}\left(t ; x_{g}\right)=\int_{B_{T}} \mathrm{~d} \nu V_{m}^{\star}(\nu) H_{r t}\left(\nu ; x_{g}\right) \mathrm{e}^{\mathrm{j} 2 \pi \nu t},
$$

where $\nu$ is the frequency variable and $B_{T}$ the bandwidth over which transient signals are measured; uppercase functions stand for Fourier spectra.

This formulation does not require to carry out cumbersome cycles of second-phase simulations for each test fault position $x_{g}$, since the transfer functions needed for locating the fault can be generated and stored for a given NUT, serving as a dictionary for decoding the position of any fault from transient detected by the probe.

For the case of a low-impedance shunt connection between two conductors, the fault-transient generator is often modeled as a unit step pulse, a choice also adopted in EMTR literature [5], representing the voltage difference between the two conductors passing instantaneously to negligible values. A more general model will be applied in this paper, assuming a finite rise time, modeling the transient as an exponential charge,

$$
v_{f}(t)=V_{o}\left(1-\exp \left(-t / T_{r}\right)\right)
$$

with a rise time $T_{r}$ and a Fourier spectrum

$$
V_{f}(\nu)=V_{o} \frac{2 \pi f_{c}}{s\left(s+2 \pi f_{c}\right)}
$$

where $s=\mathrm{j} 2 \pi \nu$ and $f_{c}=1 / 2 \pi T_{r}$. Typical values of $T_{r}$ has been reported to vary between 1 and $100 \mu$ s [13]-[16]. This kind of model will also be shown in Sec. III to help establish the feasibility of new improved metrics. Eqs. (2) and (4) show one of the pitfalls of current EMTR implementations: by using time-domain definitions, thus including low frequencies, $V_{f}(\nu)$ strongly emphasizes their contribution against that of higher frequencies. The role of $V_{f}(\nu)$ in the performance of EMTR metrics will be widely discussed in the next sections. It can already be appreciated by computing its equivalent quadratic bandwidth

$$
B_{\text {eq }}=\frac{\int_{B_{T}} \mathrm{~d} \nu \nu\left|V_{f}(\nu)\right|^{2}}{\int_{B_{T}} \mathrm{~d} \nu\left|V_{f}(\nu)\right|^{2}}=f_{1} \frac{\ln \left(f_{2} / f_{1}\right)}{1-f_{1} / f_{2}},
$$

over the bandwidth $\left[f_{1}, f_{2}\right]$, assuming $f_{2} \ll f_{c}$. As an example, for a bandwidth $[1,101] \mathrm{kHz}$, the equivalent bandwidth is about $4.7 \mathrm{kHz}$, against $100 \mathrm{kHz}$ of available data. 
The frequency-domain formulation (2) helps in this respect, since it allows to easily select a portion of the frequency data, in order to minimize the bias introduced by lower frequencies.

\section{EMTR AS A CORRELATION ESTIMATOR}

As argued in [4], [5], electrical networks in power grids are typically terminated by transformers, presenting an input impedance by far higher than the typical characteristic impedance of power lines. Therefore, each line connected to a transformer behaves as a multi-frequency resonator for transient signals, while the grid can be regarded as a set of coupled resonators. As discussed in Sec. VI, these conditions directly lead to a reverberating response, i.e., to a system with an impulse response lasting much longer than the transient generated by a fault. This kind of response is systematically found throughout the literature for fault transients.

Under these conditions, TR signals should be treated as random processes, characterized by a relatively short, but prominent, coherent part and a much longer random coda [17]-[19], orthogonal to each other. The first implication of this well-known property of TR in complex media is that the random codas do not provide any useful information, as they fundamentally behave as random Gaussian noise.

Hence, metrics based on time integration of TR signals would be inevitably affected by these noisy contributions. Only the coherent part, observed around $t=0$, can be exploited, thanks to its being practically deterministic [19]. The fact that it occurs at $t=0$ is intrinsic to the very definition of TR: this is the instant that provides the maximum coherence, with time and spatial focusing, resulting in the highest peak amplitude possible. It can therefore be expected that EMTR norms measuring peak amplitude should better perform than those based on energy: this improvement was indeed confirmed in [8].

This notwithstanding, the focus should not be on the search for the maximum peak amplitude, but rather on the fact that TR coherence only occurs at $t=0$, where the TR focused signal $v_{g}(t)$ should rather be regarded as measuring maximum coherence. In the context of fault location, having in mind how EMTR was introduced, maximum coherence is an effective measure of how close $x_{g}$, i.e., the test fault position, is to the actual one, $x_{f}$. Clearly, this property also works when assessing whether $x_{g}$ is not a good candidate for the fault position.

The point is, the $L_{\infty}$ norm selects the maximum peak occurring at any possible instant, not only at $t=0$, thus missing important information about the non-suitability of a test fault position. This discrepancy is shown in Sec. V to lead to fundamental limitations in the ability of EMTR to accurately locate faults. For these reasons, the $L_{\infty}$ norm should be substituted by the peak amplitude $P\left(x_{g}\right)$ at $t=0$, which reads

$$
\begin{aligned}
P\left(x_{g}\right) & =\int_{B_{T}} \mathrm{~d} \nu V_{m}^{\star}(\nu) H_{\mathrm{rt}}\left(\nu ; x_{g}\right) \\
& =\int_{B_{T}} \mathrm{~d} \nu V_{f}^{\star}(\nu) H_{\mathrm{dt}}^{\star}(\nu) H_{\mathrm{rt}}\left(\nu ; x_{g}\right)
\end{aligned}
$$

This norm, which is the only one that makes sense of TR self-averaging property, deserves a closer scrutiny. Indeed, (6) can be interpreted as a projection between the probe output $V_{m}(\nu)$ and simulated transfer functions $H_{\mathrm{rt}}\left(\nu ; x_{g}\right)$. In practice, it can be implemented as a simple matrix product, by arranging the $N$ test transfer functions $H_{\mathrm{rt}}\left(\nu ; x_{g}\right)$ as the rows of a matrix and the probe-recorded voltage spectrum $V_{m}(\nu)$ as a column vector, duly phase conjugated (or time reversed in time domain), i.e.,

$$
\left[\begin{array}{c}
P\left(x_{1}\right) \\
\vdots \\
P\left(x_{N}\right)
\end{array}\right]=\delta f\left[\begin{array}{c}
H_{\mathrm{rt}}\left(\nu, x_{1}\right) \\
\vdots \\
H_{\mathrm{rt}}\left(\nu, x_{N}\right)
\end{array}\right]\left[\begin{array}{c}
V_{m}^{\star}(\nu)
\end{array}\right]
$$

with $\delta f$ the step by which frequency is discretized. Eq. (7) illustrates the advantage of using a frequency-domain formulation, where $P\left(x_{g}\right)$ can be instantaneously computed once the fault transient is measured, without going through large batches of simulations meant to observe the TR focusing of transient signals.

The random nature of NUT transfer functions, as discussed in Sec. VI, affords interpreting the frequency integration as an average operator. Under these circumstances, $P\left(x_{g}\right)$ takes on a more specific meaning, namely of a cross-covariance between $V_{m}(\nu)$ and $h_{\mathrm{rt}}\left(\nu ; x_{g}\right)$. As such, it can be factorized into crosscorrelation and energy terms [20]

$$
P\left(x_{g}\right)=\rho_{V H}\left(x_{g}\right) \sqrt{\mathscr{E}_{m} \mathscr{E}_{\mathrm{rt}}\left(x_{g}\right)}
$$

with $\rho_{V H}\left(x_{g}\right)$ the cross-correlation between $V_{m}(\nu)$ and $H_{\mathrm{rt}}\left(\nu ; x_{g}\right)$, and $\mathscr{E}_{m}, \mathscr{E}_{\mathrm{rt}}\left(x_{g}\right)$ their respective mathematical energies ${ }^{1}$, with

$$
\begin{aligned}
& \mathscr{E}_{m}=\int_{B_{T}} \mathrm{~d} \nu\left|V_{m}(\nu)\right|^{2} \\
& \mathscr{E}_{\mathrm{rt}}=\int_{B_{T}} \mathrm{~d} \nu\left|H_{\mathrm{rt}}\left(\nu ; x_{g}\right)\right|^{2} .
\end{aligned}
$$

The rationale for invoking the factorization in (8) is that it points to a fundamental issue in the current implementation of EMTR. The idea behind using EMTR is to decide the position $x_{g}$ that yields the closest match between experimental and numerical results, by using focusing as a decision metric. The issue here is: what does the amplitude of $P\left(x_{g}\right)$ actually measure? Eq. (8) provides the answer to this question, by showing that it is actually a mix of two complementary contributions: on the one hand, the cross-correlation $\rho_{V H}\left(x_{g}\right)$ is a measure of similarity; on the other hand, $\mathscr{E}_{m}$ and $\mathscr{E}_{\text {rt }}\left(x_{g}\right)$ rather measure intensity. It is therefore impossible to tell whether a peak in $P\left(x_{g}\right)$ is due to having found the fault position, in which case $\rho_{V H}$ is maximum, or rather having just spotted a peak in the intensity of $H_{\mathrm{rt}}\left(x_{g}\right)$, e.g., when testing a position $x_{g}$ closer to the probe. The same observations apply to $L_{\infty}$, which was proposed in previous EMTR works. A previous attempt at using similarity-based metrics for EMTR was also recently discussed in [21], but it was rather based on the search for positions where the transient measured by the probe would be most similar to the signal observed at

\footnotetext{
${ }^{1}$ i.e., squared $L_{2}$ norms
} 
the test fault position after injecting the TR version of the transient. This approach should not be confused with the one here proposed, since it introduces a further location metric without recognizing that current EMTR metrics already are inaccurate and ineffective correlators. Indeed, [21] does not acknowledge the fundamental role of the spectrum of the transient signal, which is the main obstacle to make EMTR reliable and efficient.

The implications of highlighting the dual contributions of similarity and scale are clear: EMTR location procedures work by spotting the test position $x_{g}$ in simulation results that fits more closely those measured, thus looking for the maximum TR transmission. Scaling terms such as energies should not take part to this process, since they would readily bias comparisons of results evaluated at different test positions $x_{g}$, as indeed proven in Sec. V. In this respect, $\rho_{V H}\left(x_{g}\right)$ should be expected to ensure better results than $P\left(x_{g}\right)$, now formally defined as

$$
\rho_{V H}\left(x_{g}\right)=\frac{P\left(x_{g}\right)}{\sqrt{\mathscr{E}_{m} \mathscr{E}_{\mathrm{rt}}\left(x_{g}\right)}} .
$$

The above definition should not be confused with a posteriori normalization of the kind currently used in current EMTR implementations [5], [8]. Indeed, in (11) the normalization is meant to remove scaling factors that are intrinsically position dependent. In particular, this operation is carried out at each tested position $x_{g}$, without relying on knowledge of results obtained at other positions. Instead, standard EMTR metrics are rather based on peak-normalized results, which therefore bear a quantitative meaning only when compared to other results. This is not the case with correlation functions, since their value has a clear quantitative meaning that can be evaluated on a local level, independently from those observed at other positions. The two metrics would coincide only in case $\mathscr{E}_{\text {rt }}\left(x_{g}\right)$ were constant, i.e., independent from the position tested. In fact, Sec. V proves that $\mathscr{E}_{\mathrm{rt}}\left(x_{g}\right)$ is highly position dependent.

A direct implication of the local normalization in (11) is that by using $\rho_{V H}\left(x_{g}\right)$ it should be possible to directly decide whether a numerically tested position is a good candidate for the fault position. Before looking more closely into this property, it is useful to reformulate (11) by formalizing the interpretation of all integrals as frequency averages, introducing the averaging operator

$$
\langle g(\nu)\rangle=B_{T}^{-1} \int_{B_{T}} \mathrm{~d} \nu g(\nu),
$$

where $g(\nu)$ is a generic function. Now (11) and (6) can be restated as

$$
\rho_{V H}\left(x_{g}\right)=\frac{\left\langle V_{f}^{\star}(\nu) H_{\mathrm{dt}}^{\star}(\nu) H_{\mathrm{rt}}\left(\nu ; x_{g}\right)\right\rangle}{\sigma_{m} \sigma_{\mathrm{rt}}\left(x_{g}\right)},
$$

where $\sigma_{m}$ and $\sigma_{\mathrm{rt}}\left(x_{g}\right)$ are the (frequency-defined) standard deviations of $V_{m}(\nu)$ and $H_{\mathrm{rt}}\left(\nu ; x_{g}\right)^{2}$.

${ }^{2} \mathrm{~A}$ more accurate definition of $\sigma_{m}$ would be $\sigma_{m}^{2}=\left\langle\left|V_{m}(\nu)\right|^{2}\right\rangle-$ $\left|\left\langle V_{m}(\nu)\right\rangle\right|^{2}$, but due to the reverberant response of the NUT, $\left\langle V_{m}(\nu)\right\rangle$ can be assumed negligible. The same applies to transfer functions

\section{Mitigating the efFects of $V_{f}(\nu)$}

The values taken by $\rho_{V H}\left(x_{g}\right)$ could be easily interpreted if it were evaluated between two homogeneous quantities, e.g., two voltages: in that case their degree of similarity would be directly confirmed by a correlation close to one, as soon as $x_{g}=x_{f}$. The fact that $\rho_{V H}\left(x_{g}\right)$ rather represents a crosscorrelation makes direct interpretations less obvious since, as discussed at the end of Sec II, $V_{f}(\nu)$ is highly dispersive, thus limiting the similarity between $V_{m}(\nu)$ and $H_{\mathrm{rt}}\left(\nu ; x_{g}\right)$, even when considering $x_{g}=x_{f}$. Moreover, $V_{f}(\nu)$ in (13) disproportionately emphasizes lower frequencies, reducing the contributions from higher frequencies, resulting in a reduced effective bandwidth. It goes without saying that metrics based on correlation functions between measured and simulated probe voltages would be counterproductive, since in such a case the dispersive effects of $V_{f}(\nu)$ would be reinforced by its being squared.

The frequency-domain formulation in (6) makes it apparent that $V_{f}(\nu)$ has no useful role in assessing the similarity between numerical tested and actual responses: while in the standard implementation of TR it makes sense to inject the time-reversed signals into the same system that generated them, EMTR is actually about injecting signals into systems that are different. Reformulating this process as in (6) provides insight into the mechanisms subtending maximization of energy/peak amplitude at the fault position. In particular, from (6) it becomes apparent that similarity should be based only on transfer functions, since they completely determine the NUTs: if $H_{\mathrm{rt}}\left(\nu ; x_{f}\right)=H_{\mathrm{dt}}(\nu)$ at the fault position $x_{f}$, then $P\left(x_{f}\right)$ would be maximum only for a constant $V_{f}(\nu)$.

In view of these arguments, the accuracy of EMTR as an estimator of a fault position is expected to improve when directly evaluating the similarity between the transfer functions $H_{\mathrm{dt}}(\nu)$ and $H_{\mathrm{rt}}\left(\nu ; x_{g}\right)$, by computing their correlation $\rho_{H H}\left(x_{g}\right)$, defined as

$$
\rho_{H H}\left(x_{g}\right)=\frac{\left\langle H_{\mathrm{dt}}^{\star}(\nu) H_{\mathrm{rt}}\left(\nu ; x_{g}\right)\right\rangle}{\sigma_{\mathrm{dt}} \sigma_{\mathrm{rt}}\left(x_{g}\right)},
$$

where

$$
\sigma_{\mathrm{dt}}^{2}=\left\langle\left|H_{\mathrm{dt}}(\nu)\right|^{2}\right\rangle
$$

having adopted the formalism introduced in (13).

The problem is that the only information accessible in practice for the NUT is $V_{m}(\nu)$. If the spectrum $V_{f}(\nu)$ were known, then an inverse-filter approach could be applied to $V_{m}(\nu)$, providing an estimate of $H_{\mathrm{dt}}(\nu)$, but this option is not typically viable.

The following subsections tackle these issues by taking two different stances. In Sec. IV-A, the bias generated by a dispersive $V_{f}(\nu)$ in $\rho_{V H}\left(x_{g}\right)$ is considered, by proposing a firstorder correction that approximately reestablishes the property of $\rho_{V H}\left(x_{g}\right)$ peaking at one, without relying on an arbitrary a posteriori normalization. In Sec. IV-B, the feasibility of a blind inverse-filter procedure is analyzed, showing that it is not necessary to accurately know $V_{f}(\nu)$ in order to compensate its effects, proving under what conditions an accurate estimate of $\rho_{H H}\left(x_{g}\right)$ can be estimated. 


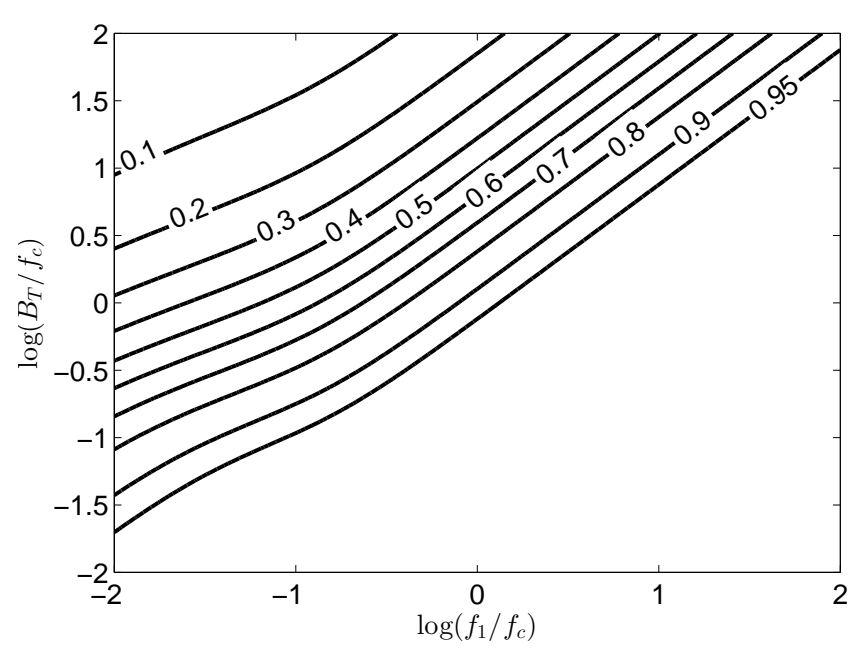

Fig. 2. Contour plot of the bias coefficient $C_{V}$, as defined in (19), as a function of the tested frequency bandwidth $\left[f_{1}, f_{2}\right]$, relative to the cut frequency $f_{c}=1 / 2 \pi T_{r}$ for an exponential step fault signal with rise time $T_{r}$.

\section{A. Cross-correlation correction}

This section studies under what conditions the crosscorrelation coefficient $\rho_{V H}\left(x_{g}\right)$ can be used as an approximate estimator of $\rho_{H H}\left(x_{g}\right)$. This is in particular useful as it provides a mean to avoid peak-normalizing results issued from EMTR processing (cf. Sec. III). As explained in Sec. III, a local normalization would make it possible to interpret results on a one-by-one basis, even before having identified the actual fault position, as otherwise required by current EMTR implementations.

To this end, the numerator of $\rho_{V H}\left(x_{g}\right)$ in (13) can be approximated as demonstrated in the Appendix, yielding

$$
\int_{B_{T}} \mathrm{~d} \nu V_{f}^{\star} H_{\mathrm{dt}}^{\star} H_{\mathrm{rt}}\left(x_{g}\right) \simeq B_{T}\left\langle V_{f}^{\star}\right\rangle\left\langle H_{\mathrm{dt}}^{\star} H_{\mathrm{rt}}\left(x_{g}\right)\right\rangle,
$$

while

$$
\sigma_{m}^{2}=\left\langle\left|V_{f}\right|^{2}\left|H_{\mathrm{dt}}\right|^{2}\right\rangle \simeq B_{T}\left\langle\left|V_{f}\right|^{2}\right\rangle \sigma_{\mathrm{dt}}^{2},
$$

which appears in the denominator of $\rho_{V H}$; a similar result applies to $\sigma_{\mathrm{rt}}^{2}\left(x_{g}\right)$. Inserting (16) and (17) into (13) results in

$$
\rho_{V H}\left(x_{g}\right) \simeq \rho_{H H}\left(x_{g}\right) C_{V},
$$

where

$$
C_{V}=\left\langle V_{f}^{\star}\right\rangle / \sqrt{\left\langle\left|V_{f}\right|^{2}\right\rangle} .
$$

Cauchy-Schwarz inequality imposes $\left|C_{V}\right| \leq 1$, with unity reached only for a constant $V_{f}(\nu)$. The strongly dispersive nature of $V_{f}(\nu)$, recalled in Sec. II, therefore leads to a bias, with $\left|C_{V}\right|<1$, confirming that the cross-correlation nature of EMTR makes its direct interpretation unwarranted, since it depends on an unknown function, i.e., $V_{f}(\nu)$.

Fig. 2 shows how $\left|C_{V}\right|$ changes depending on the frequency bandwidth over which data are collected, assuming $V_{f}(\nu)$ corresponds to the exponential step discussed in Sec. II. These results show that in order for $C_{V} \simeq 1$, it is sufficient that $B_{T} \leq f_{1}$, irrespective of the rise time $T_{r}=1 / 2 \pi f_{c}$. It is therefore not necessary to consider very narrow bandwidths

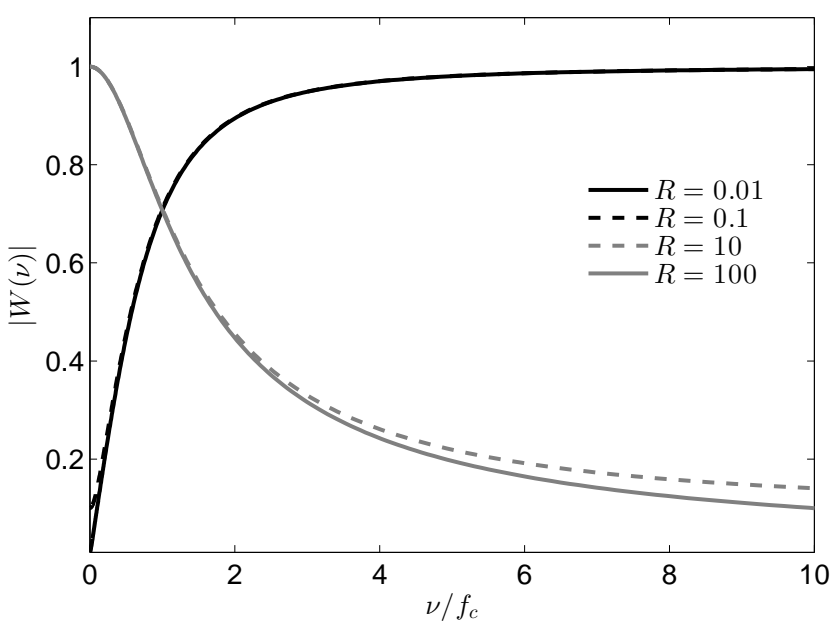

Fig. 3. Normalized modulus of $W(\nu)$ as a function of $R=\hat{f}_{c} / f_{c}$.

in order to minimize the emphasis on the low-frequency contribution. Conversely, using wide-band data, in particular when including low-frequency data, systematically results in the loss of the main advantage of correlation functions, i.e., their taking an amplitude close to one only in case of strong similarity.

\section{B. Equalization}

While choosing $B_{T}<f_{1}$ is effective in countering the most negative effects of a dispersive $V_{f}(\nu)$, it does not allow to fully benefit from all the data available. As already discussed, $V_{f}(\nu)$ being unknown, $H_{\mathrm{dt}}(\nu)$ cannot be expected to be retrievable from an inverse-filter equalization, i.e.,

$$
H_{\mathrm{dt}}(\nu)=V_{m}(\nu) / V_{f}(\nu) .
$$

In fact, it is not $H_{\mathrm{dt}}(\nu)$ that needs to be estimated accurately, but rather $\rho_{H H}\left(x_{g}\right)$. This section analyzes under what conditions the use of an approximate $\hat{V}_{f}(\nu)$ in (20) can still lead to a good estimate of $\rho_{H H}\left(x_{g}\right)$, without assuming (20).

To this end, the parametric spectrum

$$
\hat{V}_{f}(\nu)=\frac{2 \pi \hat{f}_{c}}{s\left(s+2 \pi \hat{f}_{c}\right)}
$$

will be used as an educated guess of $V_{f}(\nu)$, corresponding to the exponential step function introduced in Sec. II, with a guessed time constant $\hat{T}_{r}=1 / 2 \pi \hat{f}_{c}$. The idea is to assess under what conditions a good estimate of $\rho_{H H}\left(x_{g}\right)$ can still be expected even for $T_{r} \neq \hat{T}_{r}$.

The following estimator of $\rho_{H H}\left(x_{g}\right)$ will therefore be considered

$$
\hat{\rho}_{H H}\left(x_{g}\right)=\frac{\left\langle V_{m}^{\star}(\nu) H_{\mathrm{rt}}\left(\nu ; x_{g}\right) / \hat{V}_{f}^{\star}(\nu)\right\rangle}{\hat{\sigma}_{\mathrm{dt}} \sigma_{\mathrm{rt}}\left(x_{g}\right)},
$$

with

$$
\hat{\sigma}_{\mathrm{dt}}^{2}=\left\langle\left|V_{m}(\nu) / \hat{V}_{f}(\nu)\right|^{2}\right\rangle .
$$



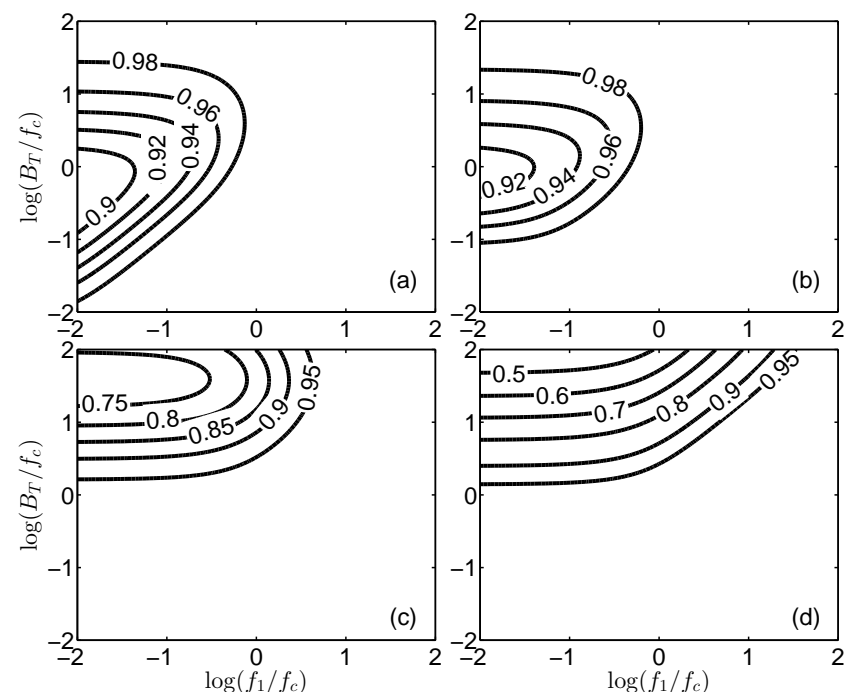

Fig. 4. Contour plots of the bias coefficient $C_{W}$, as defined in (27), as a function of the tested frequency bandwidth $\left[f_{1}, f_{2}\right]$, for four values of $R=$ $\hat{f}_{c} / f_{c}$ : (a) 0.01, (b) 0.1, (c) 10 and (d) 100 .

Approximating the spectrum of $V_{f}(\nu)$ as in (4), (22) can be restated as

$$
\hat{\rho}_{H H}\left(x_{g}\right)=\frac{\left\langle H_{\mathrm{dt}}^{\star}(\nu) H_{\mathrm{rt}}\left(\nu ; x_{g}\right) W(\nu)\right\rangle}{\sigma_{\mathrm{rt}}\left(x_{g}\right) \sqrt{\left\langle\left|H_{\mathrm{dt}}(\nu)\right|^{2}|W(\nu)|^{2}\right\rangle}},
$$

where

$$
W(\nu)=\frac{s+2 \pi \hat{f}_{c}}{s+2 \pi f_{c}}=\frac{\mathrm{j} \nu / f_{c}+\hat{f}_{c} / f_{c}}{\mathrm{j} \nu / f_{c}+1}
$$

is a weighting function that measures the residue of an imperfectly uncompensated $V_{f}(\nu)$ after the equalization.

From (25) it appears that the flatness of $W(\nu)$ depends on the relative position of its pole and zero. More precisely, if (24) were evaluated at frequencies $\nu<\min \left(f_{c}, \hat{f}_{c}\right)$, or $\nu>$ $\max \left(f_{c}, \hat{f}_{c}\right), W(\nu)$ would still be relatively flat, as visible from Fig. 3, resulting in an accurate estimate of $\rho_{H H}\left(x_{g}\right)$. The scenarios where $\hat{f}_{c}<f_{c}$ result in a flatter $|W(\nu)|$.

In general, an imperfect correction of $V_{f}(\nu)$ has $W(\nu) \neq 1$ and would therefore result in $\hat{\rho}_{H H}\left(x_{f}\right)<1$. The extent of this effect can be quantified by applying to (24) the same approximation used in (16) and (17) for deriving (19), yielding

$$
\hat{\rho}_{H H}\left(x_{g}\right) \simeq \rho_{H H}\left(x_{g}\right) C_{W},
$$

with

$$
C_{W}=\langle W(\nu)\rangle / \sqrt{\left\langle|W(\nu)|^{2}\right\rangle} .
$$

Since $W(\nu)$ is substantially flatter than $V_{f}(\nu), C_{W}$ is expected to be closer to one than $C_{V}$. Fig. 4 shows how $C_{W}$ changes for a broad set of scenarios, with the ratio $R=\hat{f}_{c} / f_{c}$ changing from 1/100 to 100. As argued above, the case $R \leq 1$ yields the more robust results, with $C_{W}$ taking values above 0.9 in all cases but when using data acquired at very low frequencies, much smaller than $f_{c}$. The minimum value taken by $C_{W}$ in this case can be evaluated from (27) to be equal to $\sqrt{3} / 2 \simeq 0.86$. Conversely, if $R>1, C_{W}$ is significantly smaller than one only if data cover a large bandwidth, reaching out the transition region between $f_{c}$ and $\hat{f}_{c}$, shown in Fig. 3 .
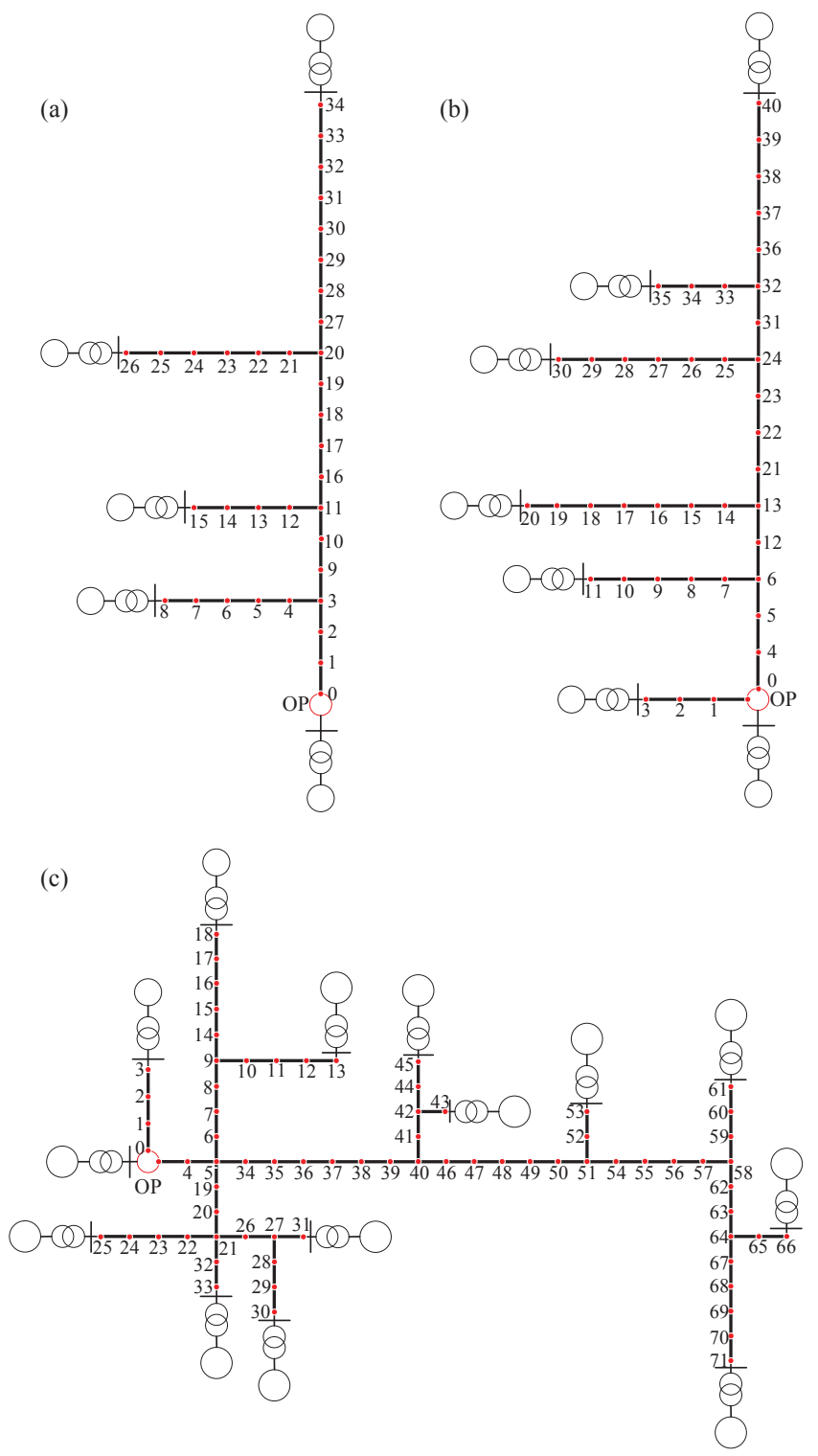

Fig. 5. Schematics for the three grid structures considered in the paper, for: (a) three-junction, (b) five-junction and (c) ten-junction distribution networks. Numerical tags identify the fault positions tested in Sec. V, with $1 \mathrm{~km}$ separating each section between two tags. The grids are terminated by transformers. The voltage probe monitoring fault transients is found at tag no. 0 , for all three grids.

Overall, Fig. 4 confirms the feasibility of a blind inverse filtering, suggesting to set the value of $f_{c}$ equal to the lower bound of typical values expected for $f_{c}$. This property should not be read as an invitation to choose very small values of $\hat{f}_{c}$ : for the sake of equalization, this ratio should be as close as possible to one. It should rather be seen as a requirement not to choose $\hat{f}_{c} / f_{c} \gg 1$. It is worth noticing that these results prove that the actual value of $\hat{f}_{c}$ is not important for the application of (26), unless gross errors are made. The relative insensitivity of $\hat{\rho}_{H H}\left(x_{g}\right)$ to the choice of $\hat{f}_{c}$, as long as $\hat{f}_{c} / f_{c} \ll 1$ is confirmed in Sec. V. 

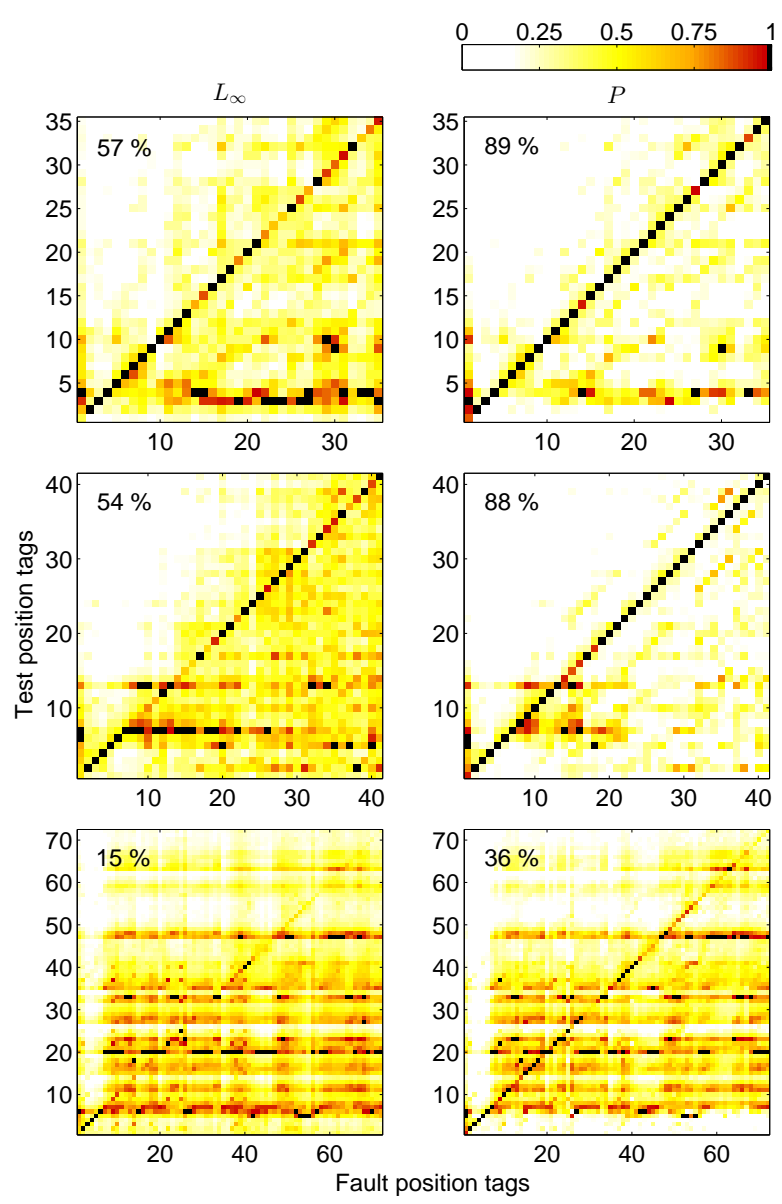

Fig. 6. Comparison of EMTR location accuracy using $L_{\infty}$ norm (left column) and $P$ norm (right column), which is restricted to $t=0$. Results for three NUTs in Fig. 5, from top to bottom, computed over the frequency range $[10-1000] \mathrm{kHz}$

\section{Simulation RESUlts}

This section compares the performance of each of the norms discussed so far, when applying the EMTR location criterion (1) to the three networks shown in Fig. 5. Several goals guided the definition of the comparisons: 1) the network complexity, measured in terms of the number of junctions, 2) the relative position and distance between the probe and the fault, 3) the effects of the frequency-response dynamics by using data from a selected bandwidth rather than the entire dataset available.

Each network consists of uniform lines where losses are assumed to be negligible. The rationale for this simplification is to avoid any spurious effect due to losses, which could mask differences due to the metrics themselves. Each line is terminated by either a junction or a transformer. As already discussed in [5], the transformers are assumed to present highimpedance conditions, therefore regarding each line terminated by transformers as highly reflecting; a $10 \mathrm{k} \Omega$ equivalent load is used for the sake of accounting for potential losses in the transformers. The probe used for monitoring the occurrence of fault transient signals is here modeled by measuring the voltage induced at the terminations with tag no. 0 , shown in Fig. 5. Only single-phase lines are considered, since the ability of EMTR to discriminate between different phases was already well established in [5].

The propagation of fault-induced signals along these three distribution networks was simulated using EMTP-RV, by modeling the fault using an equivalent Thevenin circuit, with a voltage generator $v_{f}(t)$ representing the voltage transient (cf. Fig. 1(a)), with a fault impedance $Z_{F}=10 \Omega$, thus much smaller than the typical characteristic impedance of the lines. This last choice, which is consistent with the idea of shunt faults, also leads to high-reflectivity conditions. A large number of positions for the fault were considered, by moving it along every line in the three NUTs, with a spatial step of $1 \mathrm{~km}$. The rationale is to assess whether EMTR locates faults with the same accuracy independently from their distance from the probe. Numerical tags in Fig. 5 allow to identify the position of each fault studied. For each fault position, a simulation was run, covering a maximum frequency of $1 \mathrm{MHz}$. The fault equivalent generator $v_{f}(t)$ was modeled as discussed in Sec. II, as an exponential transient with a typical rise time of $10 \mu \mathrm{s}$.

Transient voltages $v_{m}(t)$ were computed with EMTP-RV for each fault position. All the metrics discussed in Secs. II to IV generate results for every position $x_{g}$, with tags given in Fig. 5, peaking at a position $\hat{x}_{f}$, ideally corresponding to the fault position $x_{f}$. Due to the sheer number of combinations of fault positions $x_{f}$ and test fault positions $x_{g}$, a compact matrix representation was chosen, instead of standard cartesian plots, with columns and rows referring to $x_{f}$ and $x_{g}$, respectively. This choice allows to easily spot an ideal location estimator, since it would present a diagonal standing out from a lower background.

Before going through the overall comparison of metrics, the argument presented in Sec. II pushing for limiting EMTR metrics to be only based on the peak amplitude at $t=0$ are validated in Fig. 6, by comparing the metrics issued from $L_{\infty}\left(x_{g}\right)$ and $P\left(x_{g}\right)$ norms, for a frequency bandwidth $[10,1000] \mathrm{kHz}$. These results prove that metrics looking for the peak amplitude at $t \neq 0$ significantly increase the risk of incorrect fault location, with $P\left(x_{g}\right)$ featuring a rate of accurate location up to twice that of the $L_{\infty}$ norm. The main reason for the poorer performance of $L_{\infty}$ is that EMTR does not correspond to standard TR, where time-reversed signals are injected back into the same system that generated them in the first place. EMTR expects signal focusing to be maximized when the test fault position $x_{g}=x_{f}$, consistently with standard TR. But in case $x_{g} \neq x_{f}$, there is no way of ensuring that $L_{\infty}\left(x_{g}\right)<L_{\infty}\left(x_{f}\right), \forall t \neq 0$, since the two associated NUTs will be different in this case. Moreover, only at $t=0$ standard TR would lead to focusing or, in other terms, to maximal coherence and therefore minimum dependence from the characteristics of a NUT.

Fig. 6 clearly show the appearance of wrong fault positions with $L_{\infty}$, i.e., outside the main diagonal, corresponding in the vast majority of cases to branches close to the one containing the actual fault. Limiting the $L_{\infty}$ metric to $t=0$, i.e., switching to $P$, effectively reduces the number of such instances; each plot in Fig. 6 reports the percentage of fault positions accurately located by the two norms. The risk of an erroneous location appears to significantly depend on the 

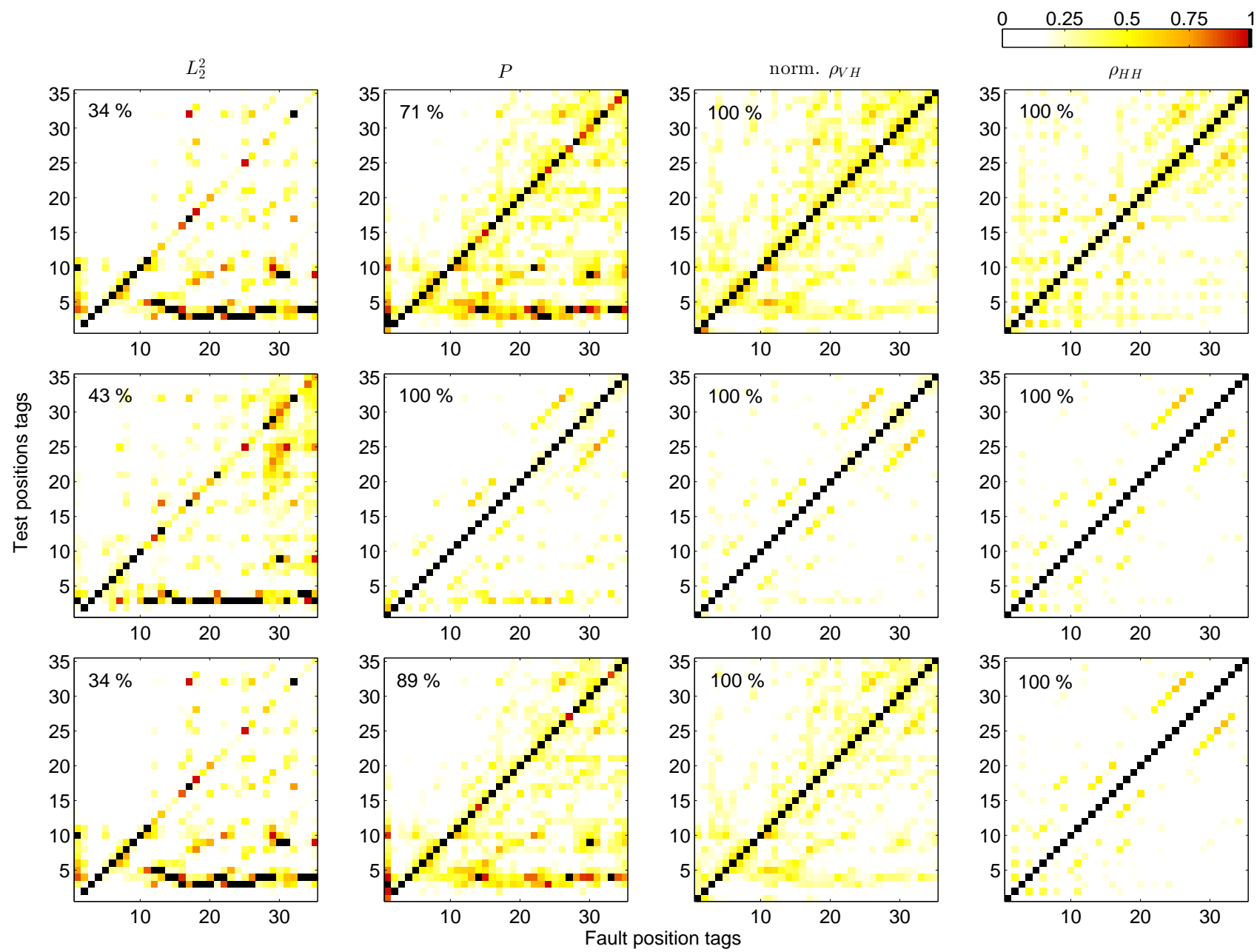

Fig. 7. Performance of the four EMTR metrics applied to the three-junction network in Fig .5(a), for three different frequency bandwidths: $10-100 \mathrm{kHz}$ (top row), $100-1000 \mathrm{kHz}$ (middle row) and $10-1000 \mathrm{kHz}$ (bottom row). The rate of accurate location is shown on each graph. Results for $\rho_{V H}$ are normalized in order to simplify their comparison.

network complexity, for $L_{\infty}$, while $P$ is less sensitive to it. This observation implies that as a network becomes more complex, the probability of appearance of spurious peaks in time-reversed signals increases. Following these observations, the $P$ norm will be used instead of the $L_{\infty}$ norm.

Fig. 7 presents a thorough analysis of the performance of four metrics: 1) $L_{2}^{2}$, 2) $P$, 3) $\rho_{V H}$ and 4) $\rho_{H H}$. The first observation is that the $L_{2}^{2}$ norm has the worst performance, with a large number of erroneous fault locations. As discussed in Sec. III, when refocusing on the fault position, timereversed signals produce two contributions: a coherent one, associated to the largest amplitude peak and typically weaker background fluctuations, with a much longer duration that depends on the reverberation decay time of the NUT (cf. Sec. VI). Therefore, since $L_{2}^{2}$ requires integrating the entire signal intensity, background fluctuations, even though locally weaker than the coherent part, have a non-negligible contribution. Since they are by definition orthogonal to the coherent part [19], they do not provide useful information about the fault position, and rather behave as random noise.

The norm $P$ can be expected to perform better, since it only uses the coherent contribution, reaching its peak at $t=0$, which is the best approximation of the original fault transient signal, in a matched-filter sense. The rate of correct location is indeed much higher than for the $L_{2}^{2}$ norm. Yet, $L_{2}^{2}$ and $P$ share another kind of error: as faults get farther away from the probe, faults location may be erroneously estimated to be at a closer distance from the probe. This phenomenon is observed in Fig. 7, where faults with a position tag above 10 are wrongly located mainly at position tag 3 , otherwise at 4,5 and 9 . The most likely reason for these errors is the fact that $L_{2}^{2}, P$ and also $L_{\infty}$ norms cannot separate similarity and scale contributions, as discussed in Sec. III. In practical terms, time-reversed signals do focus on the fault position, but as soon as signals need to propagate across multiple junctions, their intensity weakens, potentially resulting in a lower energy or peak amplitude with respect to other positions tested. Indeed, passing to locally normalized estimators, such as $\rho_{V H}$, all but totally removes spurious locations, resulting in an ideal location performance. Scale effects due to propagation attenuation driven by junctions, discussed at the end of this section, confirm this interpretation. 

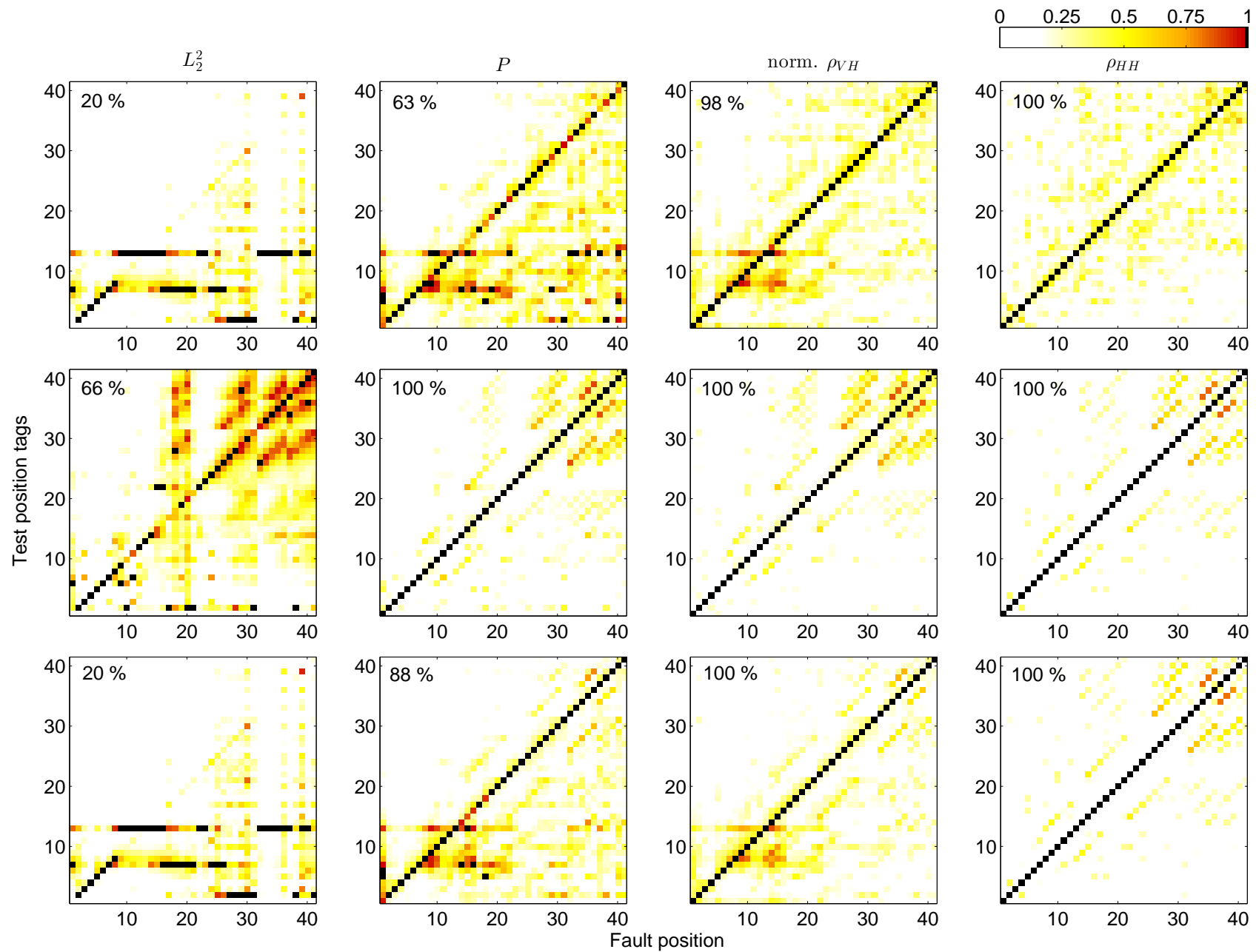

Fig. 8. Performance of the four EMTR metrics applied to the five-junction network in Fig .5(b), for three different frequency bandwidths: 10-100 kHz (top row), $100-1000 \mathrm{kHz}$ (middle row) and $10-1000 \mathrm{kHz}$ (bottom row). The rate of accurate location is shown on each graph. Results for $\rho_{V H}$ are normalized in order to simplify their comparison.

The last norm tested, $\rho_{H H}$, has a performance similar to $\rho_{V H}$ for the NUT in Fig. 5(a). The main difference is in the strong reduction of residual local maxima that could potentially be interpreted as alternative fault locations. The results in Fig. 7 for $\rho_{V H}$ present several such maxima, in the 0.7-0.8 range, which should not be neglected, in particular in case of data affected by noise, occurring less frequently for $\rho_{H H}$.

Results in Fig. 7 were computed for three different choices of frequency bandwidths, in order to illustrate how frequency selection affects location accuracy. It can be noticed how fault location improves when passing from a bandwidth $10-100$ $\mathrm{kHz}$ to $100-1000 \mathrm{kHz}$, for all four metrics considered. In particular, spurious maxima become less intense and likely. Conversely, extending tests at lower frequencies, for a bandwidth $10-1000 \mathrm{kHz}$, results in $L_{2}^{2}, P$ and $\rho_{V H}$ performing worse than for a $100-1000 \mathrm{kHz}$, with results very similar to those obtained for the $10-100 \mathrm{kHz}$ bandwidth. This issue apparently does not occur for $\rho_{H H}$. The reason for this behavior is found in the spectrum of the fault transient, $V_{f}(\nu)$, which highly emphasizes lower frequencies to the expense of high-frequency information, as discussed in Secs. II and III. In practice, depending on the choice of bandwidth for the tests, the highly dispersive nature of $V_{f}(\nu)$ can strongly undermine EMTR performance, but for $\rho_{H H}$, which is ideally independent from $V_{f}(\nu)$. These results confirm the practical interest of mitigating the effects of $V_{f}(\nu)$ in EMTR metrics, in order to benefit from the whole available bandwidth. Previous implementations of EMTR were based on a time-domain description [5], [8], thus even more strongly sensitive to the fault spectrum $V_{f}(\nu)$, since in this case no attempt was made in reducing the dominance of low-frequency contributions.

In practical terms, the results in Fig. 7 also demonstrate that there is no need to consider wide-band tests in order for EMTR to accurately locate faults, as locally-normalized metrics $\rho_{V H}$ and $\rho_{H H}$ outperform $L_{2}^{2}, L_{\infty}$ and $P$ even when testing below $100 \mathrm{kHz}$.

The effects of an increased complexity in the NUT can be appreciated from results in Figs. 8 and 9. The same conclusions described for the three-junction NUT are also reached for these two further cases. But as the complexity increases, the number and intensity of spurious peaks follow 

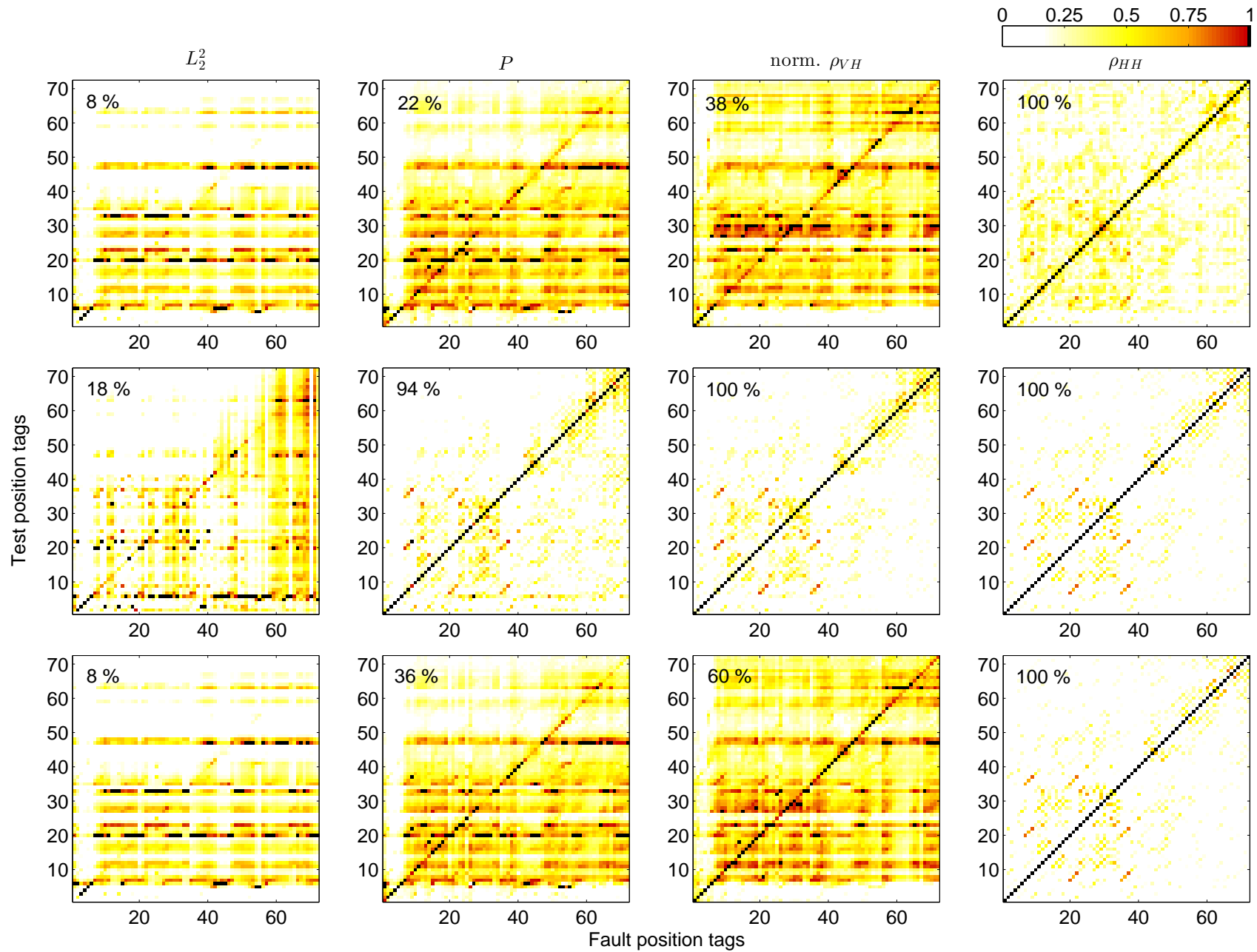

Fig. 9. Performance of the four EMTR metrics applied to the ten-junction network in Fig .5(c), for three different frequency bandwidths: $10-100 \mathrm{kHz}$ (top row), $100-1000 \mathrm{kHz}$ (middle row) and $10-1000 \mathrm{kHz}$ (bottom row). The rate of accurate location is shown on each graph. Results for $\rho_{V H}$ are normalized in order to simplify their comparison.

suit, posing a higher risk of erroneous fault location. The performance of $L_{2}^{2}$ and $P$ norms is strongly affected, with location rates as low as $8 \%$, in particular when using data extending over several decades. Results for $P$ are much less affected when working on a flatter portion of $V_{f}(\nu)$, e.g., 100$1000 \mathrm{kHz}$.

The results for the five- and ten-junction cases clearly show that even $\rho_{V H}$ becomes increasingly sensitive to the choice of test bandwidth, as opposed to $\rho_{H H}$, which is $100 \%$ accurate in all tested cases. Again, the negative effects of $V_{f}(\nu)$ explain the worsening performance for $\rho_{V H}$, supporting the proposal presented in Sec. IV-B for a blind inverse filtering of the fault transient recorded by the probe.

All the results pertaining to $\rho_{H H}$ in Figs. 7 to 9 were computed by assuming $V_{f}(\nu)$ known. In practice, such assumption may not hold, and a blind inverse filtering would be necessary, as discussed in Sec. IV-B. Fig. 10 shows how the estimated $\hat{\rho}_{\mathrm{HH}}$ changes when using a guess $\hat{V}_{f}(\nu)$ with a wide variation of choices for a guess rise time $\hat{T}_{r}$, from $0.1 \mu \mathrm{s}$ to $1 \mathrm{~ms}$, while the actual fault transient had a $10 \mu$ s rise time. As predicted by Fig. 4 , for $\hat{T}_{r} / T_{r} \geq 1$, the estimated $\hat{\rho}_{\mathrm{HH}}$ is weakly dependent from the actual choice of $\hat{T}_{r}$, even when $\hat{T}_{r} / T_{r} \gg 1$. Conversely, as $\hat{T}_{r} / T_{r} \ll 1$ the main effect is that $\hat{\rho}_{\mathrm{HH}}$ may become significantly smaller than one even over the fault position. This last scenario could be avoided by a posteriori normalization, as in previous work on EMTR norms [8], but such an approach would result in the loss of quantitative interpretation of $\hat{\rho}_{\mathrm{HH}}$, i.e., the ability to decide at any test position $x_{g}$, whether it represents a good candidate for the fault position, without relying on any comparison with other test positions.

Finally, Sec. III suggested that the main reason for the appearance of spurious peaks in $L_{2}^{2}$ and $P$ norms is the non-uniform spatial distribution of the intensity of transfer functions, depending on the position of a fault with respect to the probe. Fig. 11 shows this distribution for the three NUTs, confirming that transfer functions see their intensity broadly reducing when moving through a junction. Therefore, faults more deeply embedded into a NUT will be characterized by a lower focusing energy/amplitude, fostering the conditions for spurious fault locations. 

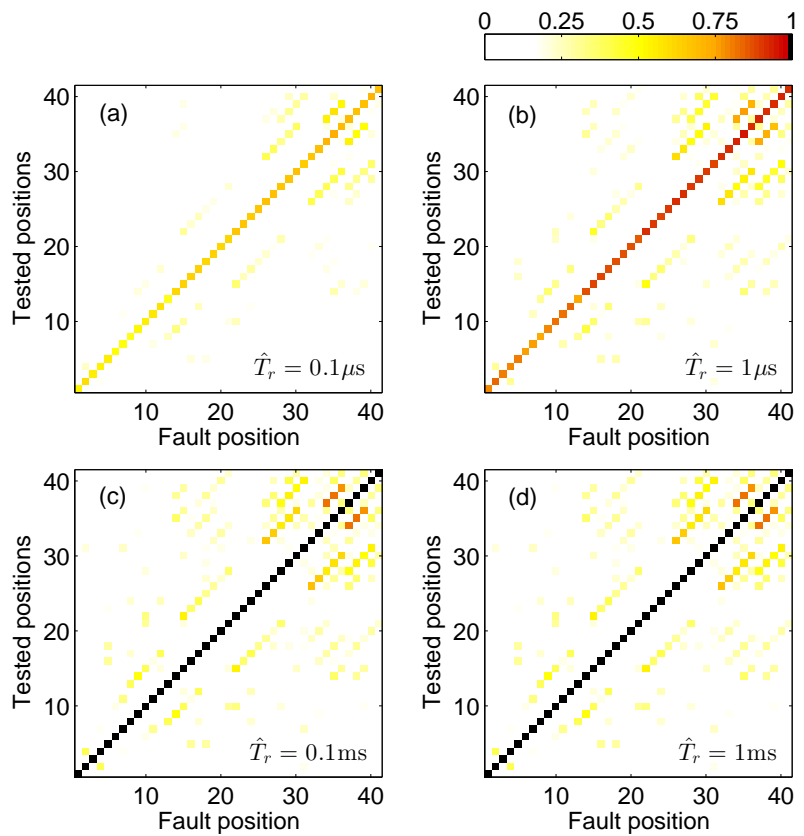

Fig. 10. Estimates $\hat{\rho}_{\mathrm{HH}}$ of $\rho_{H H}$ obtained from blind inverse filtering of $V_{m}(\nu)$ for four guessed values of $\hat{T}_{r}$, as computed for the five-junction NUT in Fig. 5(b), for an actual $T_{r}=10 \mu \mathrm{s}$.

\section{REVERBERATION, RELAXATION TIME AND MINIMUM BANDWIDTH REQUIREMENTS FOR EMTR}

This section discusses under what conditions EMTR-based location metrics can be expected to be reliable. By this terms, it is meant the property of EMTR to generate a coherent focusing, thus ideally deterministic. To this end, it is necessary to recall that Sec. III argued that a power grid with highimpedance terminations is basically a collection of coupled resonators. An example of transfer functions shown in Fig. 12, for the five-junction network, effectively confirms this expectation: a large dynamic range spanned by the transfer functions, with a maximum-to-minimum ratio exceeding 100 , is a clear indication of a strong resonant behavior.

Time-domain statistics such as the power-delay profile ${ }^{3}$ shown in Fig. 13 confirm that the three NUTs in Fig. 13 are more than resonant, as they are actually reverberating, with a relaxation (or decay) time $\tau$ just above $0.55 \mathrm{~ms}$. With a typical length of roughly $20 \mathrm{~km}$, the propagation delay for a signal to cross these networks is about $60 \mu \mathrm{s}$, implying that signals reverberate through the networks hundreds of times before dissipating.

Being highly reverberant systems, the necessary conditions for TR to be reliable were derived and verified in [19], [22] to require that the ratio $B_{T} / B_{c} \gg 1$, with $B_{c} \simeq 1 / \tau$ the coherence time of the medium, here power grids. Hence, a minimum bandwidth requirement reads

$$
B_{T} \gg 1 / \tau,
$$

i.e., $B_{T} \gg 1.8 \mathrm{kHz}$ for the three networks studied in this paper. The ratio $B_{T} / B_{c}$ measures the number of degrees

${ }^{3}$ defined as the average of the squared impulse responses of a system

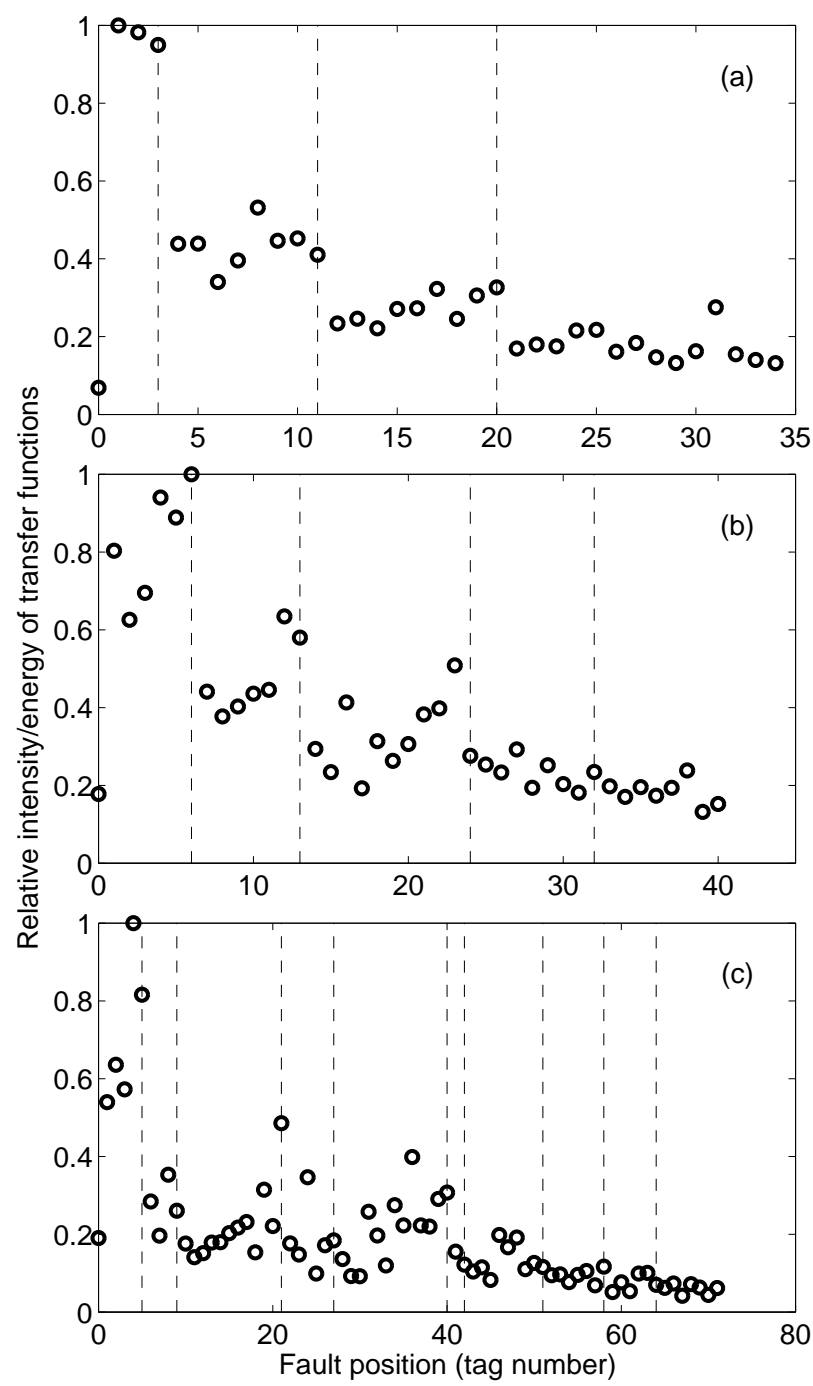

Fig. 11. Normalized energy transmission associated to the transfer functions of each fault position, computed over the frequency range $[10,1000] \mathrm{kHz}$, for the three NUTs in Fig. 5. Vertical dashed lines show the position of junctions.

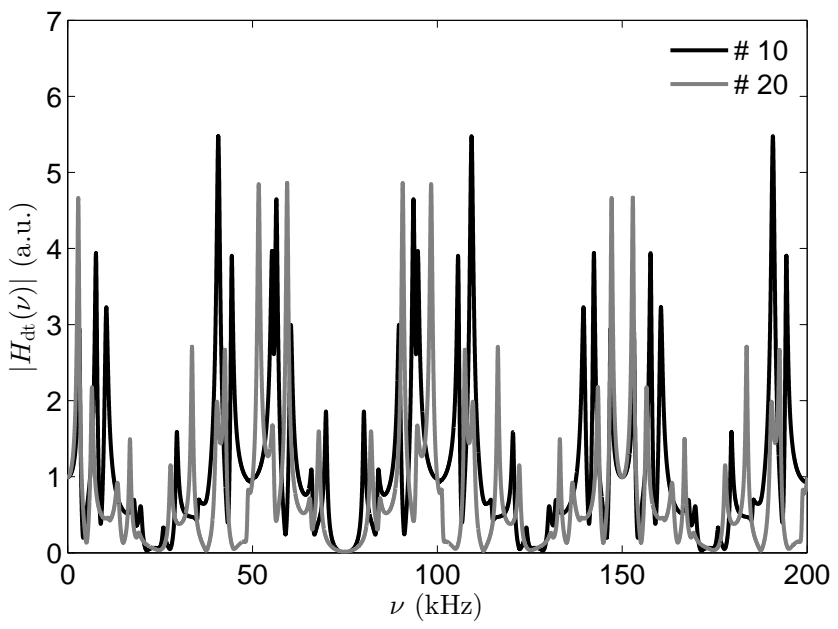

Fig. 12. An example of transfer functions for the five-junction NUT in Fig. $5(\mathrm{~b})$, as observed at the two fault positions shown in the figure. 


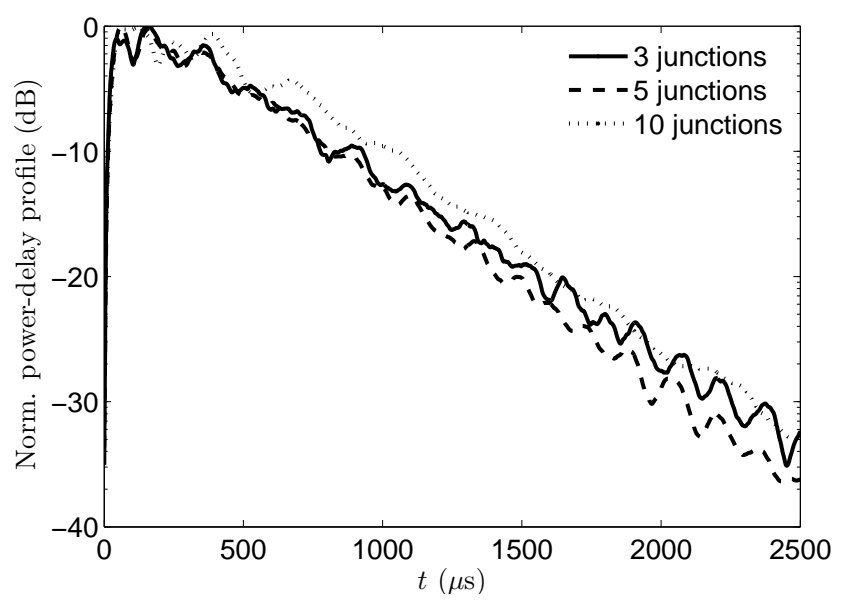

Fig. 13. Power-delay profiles for the three NUT in Fig. 5.
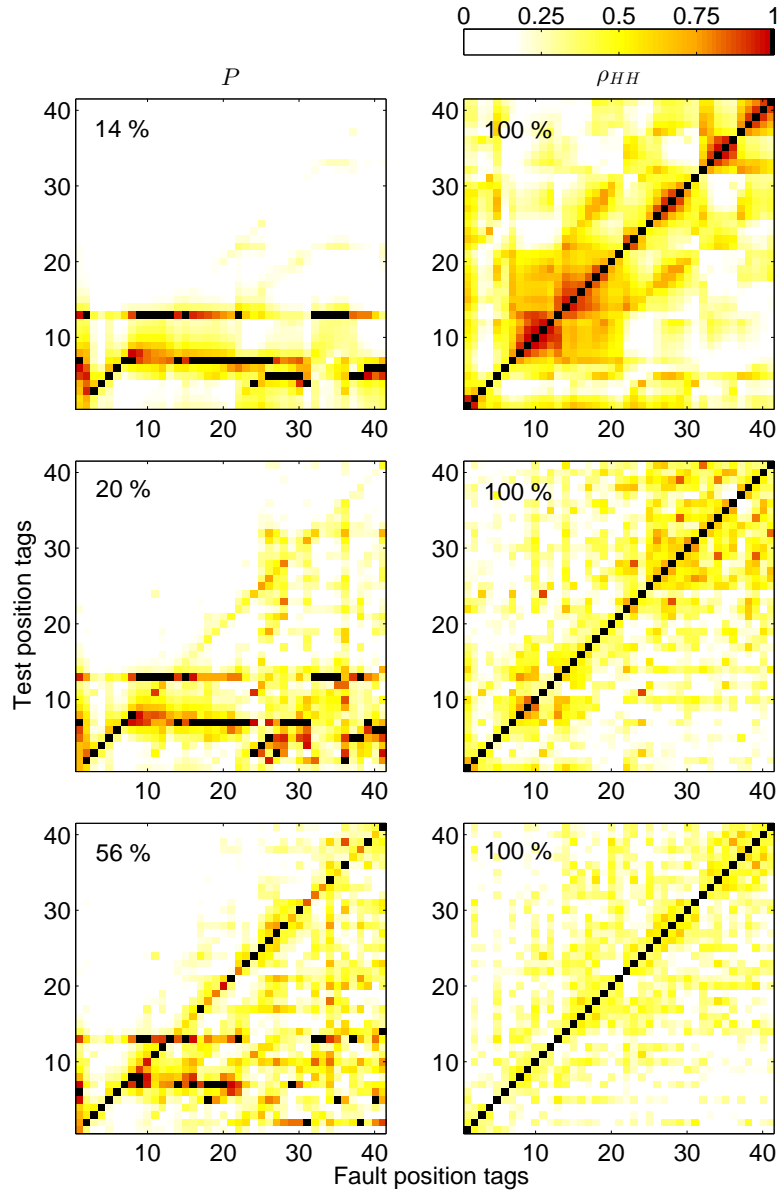

Fig. 14. Self averaging in action: convergence of $P$ and $\rho_{H H}$ norms for the five-junction NUT in Fig. 5(b), for $f_{1}=10 \mathrm{kHz}$ and $B_{T}$ equal to : a) 15 $\mathrm{kHz}$ (top row), b) $30 \mathrm{kHz}$ (middle row) and c) $60 \mathrm{kHz}$ (bottom row).

of freedom, or independent information collected across the bandwidth $B_{T}$; these grains of information underlie the most important property of TR, i.e., self averaging [19], [23], [24].

Fig. 14 shows a clear example of self averaging, where the location accuracy of $P$ and $\rho_{H H}$ norms improve as $B_{T}$ increases. Notice how $\rho_{H H}$ can already provide reliable results even at $B_{T}=15 \mathrm{kHz}$, for which $B_{T} / B_{c} \simeq 8.3$, i.e., below the limit of validity of (28), thus showing signs of loss of location accuracy in the shape of extended regions around the actual fault position where even $\rho_{H H}$ is no longer accurate. The much poorer performance of $P$ is explained by a reduced effective bandwidth due to the low-frequency emphasis of $V_{f}(\nu)$, with (28) only partially met.

\section{CONCLUSION}

This paper has investigated how EMTR performs in case of complex power-distribution networks, with a particular attention at how the relative position of the fault with respect to the probe affects the results. It has confirmed that current metrics based on energy and peak-amplitude focusing may present a non-negligible bias caused by the non-uniform distribution of high-frequency electrical energy throughout complex networks, as excited by the fault.

The reformulation of the EMTR processing in the frequency domain has led to an original interpretation of EMTR as a correlation estimator, highlighting the reasons for potential failure with current energy and peak-amplitude metrics. Two local normalization approaches were proposed, in order to take into account the non-uniform propagation of transient-signal energy in complex networks. These new metrics reinstate quantitative reading of EMTR results, without relying on a posteriori peak normalization, which would be intrinsically dependent on the tested fault positions. Both new metrics were shown to dramatically reduce the occurrence of erroneous fault location.

In particular, the blind inverse filtering behind $\hat{\rho}_{\mathrm{HH}}$ was confirmed as very effective in exploiting all the information available from transient signals, systematically yielding $100 \%$ correct estimates of the fault position, even when deeply embedded into complex networks. Its remarkably low sensitivity to the characteristics of the fault transient makes it a promising location criterion, even more so considering that it strongly reduces the need for large bandwidths appearing in previous EMTR work, an important feature when dealing with realistic distribution lines that may significantly attenuate higher frequencies.

\section{APPENDIX}

Given a smooth function $S(\nu)$ and a random process $R(\nu)$, with a coherence bandwidth $B_{c}$, the integral

$$
\mathscr{I}=\int_{f_{1}}^{f_{2}} \mathrm{~d} \nu S(\nu) R(\nu)
$$

can be computed by first solving it by parts

$$
\mathscr{I}=\left[S(\nu) C_{R}(\nu)\right]_{f_{1}}^{f_{2}}-\int_{f_{1}}^{f_{2}} \mathrm{~d} \nu S^{\prime}(\nu) C_{R}(\nu),
$$

where $S^{\prime}(\nu)$ is the first derivative of $S(\nu)$ and $C_{R}(\nu)$ a primitive of $R(\nu)$, and

$$
C_{R}(\nu)=\int_{f_{1}}^{\nu} \mathrm{d} \xi R(\xi)=\bar{R}(\nu)\left(\nu-f_{1}\right),
$$


with $\bar{R}(\nu)$ the average value of $R(\xi)$ over the frequency range $\left[f_{1}, \nu\right]$. This reformulation of (29) has an important advantage: by introducing the average $\bar{R}(\nu)$, as soon as

$$
\left(\nu-f_{1}\right) \gg B_{c},
$$

$\bar{R}(\nu)$ will closely approximate its average value $\langle R(\nu)\rangle$ computed over the frequency range $\left[f_{1}, f_{2}\right]$, as defined in (12). Since applications of TR to complex media call for the condition (32) as necessary (cf. Sec. VI), $\bar{R}(\nu) \simeq\langle R(\nu)\rangle$.

Considering this approximation, (30) can be recast as

$$
\mathscr{I} /\langle R(\nu)\rangle=S\left(f_{2}\right)\left(f_{2}-f_{1}\right)-\int_{f_{1}}^{f_{2}} \mathrm{~d} \nu S^{\prime}(\nu)\left(\nu-f_{1}\right),
$$

and developed into

$$
\mathscr{I} /\langle R(\nu)\rangle=\left[S\left(f_{2}\right) f_{2}-S\left(f_{1}\right) f_{1}\right]-\int_{f_{1}}^{f_{2}} \mathrm{~d} \nu \nu S^{\prime}(\nu) .
$$

The last integral can be integrated by part,

$$
\begin{aligned}
\int_{f_{1}}^{f_{2}} \mathrm{~d} \nu \nu S^{\prime}(\nu) & =\left[S\left(f_{2}\right) f_{2}-S\left(f_{1}\right) f_{1}\right]-\int_{f_{1}}^{f_{2}} \mathrm{~d} \nu S(\nu) \\
& =\left[S\left(f_{2}\right) f_{2}-S\left(f_{1}\right) f_{1}\right]-\langle S(\nu)\rangle\left(f_{2}-f_{1}\right),
\end{aligned}
$$

with $\langle S(\nu)\rangle$ the average value of $S(\nu)$ over $\left[f_{1}, f_{2}\right]$.

Substituting (35) into (34) yields

$$
\mathscr{I}=\int_{f_{1}}^{f_{2}} \mathrm{~d} \nu S(\nu) R(\nu) \simeq\langle S(\nu)\rangle\langle R(\nu)\rangle B_{T},
$$

with $B_{T}=f_{2}-f_{1}$.

\section{REFERENCES}

[1] P. F. Gale, P. A. Crossley, X. Bingyin, G. Yaozhong, B. J. Cory, and J. R. G. Barker, "Fault location based on travelling waves," in 1993 Fifth International Conference on Developments in Power System Protection, March 1993, pp. 54-59.

[2] G. B. Ancell and N. C. Pahalawaththa, "Maximum likelihood estimation of fault location on transmission lines using travelling waves," IEEE Transactions on Power Delivery, vol. 9, no. 2, pp. 680-689, April 1994.

[3] A. O. Ibe and B. J. Cory, "A travelling wave-based fault locator for twoand three-terminal networks," IEEE Transactions on Power Delivery, vol. 1, no. 2, pp. 283-288, April 1986.

[4] Z. Q. Bo, G. Weller, and M. A. Redfern, "Accurate fault location technique for distribution system using fault-generated high-frequency transient voltage signals," IEE Proceedings - Generation, Transmission and Distribution, vol. 146, no. 1, pp. 73-79, Jan 1999.

[5] R. Razzaghi, G. Lugrin, H. Manesh, C. Romero, M. Paolone, and F. Rachidi, "An efficient method based on the electromagnetic time reversal to locate faults in power networks," IEEE Transactions on Power Delivery, vol. 28, no. 3, pp. 1663-1673, 2013.

[6] M. Fink, "Time reversal of ultrasonic fields. I. Basic principles," IEEE Transactions on Ultrasonics, Ferroelectrics and Frequency Control, vol. 39, no. 5, pp. 555-566, 1992.

[7] M. Fink, D. Cassereau, A. Derode, C. Prada, P. Roux, M. Tanter, J. Thomas, and F. Wu, "Time-reversed acoustics," Reports on Progress in Physics, vol. 63, p. 1933, 2000.

[8] S. He, Y. Xie, Z. Wang, F. Rachidi, B. Liu, Q. Li, and X. Kong, "Norm criteria in the electromagnetic time reversal technique for fault location in transmission lines," IEEE Transactions on Electromagnetic Compatibility, vol. 60, no. 5, pp. 1240-1248, Oct 2018.

[9] R. Razzaghi, M. Paolone, F. Rachidi, J. Descloux, B. Raison, and N. Retière, "Fault location in multi-terminal hvdc networks based on electromagnetic time reversal with limited time reversal window," in 2014 Power Systems Computation Conference, Aug 2014, pp. 1-7.
[10] R. Razzaghi, G. Lugrin, F. Rachidi, and M. Paolone, "Assessment of the influence of losses on the performance of the electromagnetic time reversal fault location method," IEEE Transactions on Power Delivery, vol. 32, no. 5, pp. 2303-2312, Oct 2017.

[11] Z. Wang, S. He, Q. Li, B. Liu, R. Razzaghi, M. Paolone, Y. Xie, M. Rubinstein, and F. Rachidi, "A full-scale experimental validation of electromagnetic time reversal applied to locate disturbances in overhead power distribution lines," IEEE Transactions on Electromagnetic Compatibility, vol. 60, no. 5, pp. 1562-1570, Oct 2018 .

[12] A. Greenwood, Electrical transients in power systems. John Wiley and Sons Inc., New York, USA, 1991.

[13] A. Ametani, T. Ono, Y. Honaga, and Y. Ouchi, "Surge propagation on japanese $500 \mathrm{kv}$ untransposed transmission line," Proceedings of the Institution of Electrical Engineers, vol. 121, no. 2, pp. 136-138, February 1974.

[14] T. Cui, X. Dong, Z. Bo, and A. Juszczyk, "Hilbert-transform-based transient/intermittent earth fault detection in noneffectively grounded distribution systems," IEEE Transactions on Power Delivery, vol. 26, no. 1, pp. 143-151, Jan 2011.

[15] T. Ohtaka, M. Iwata, S.-i. Tanaka, and Y. Goda, "Development of an emtp simulation model of arcing horns interrupting fault current," IEEE Transactions on Power Delivery, vol. 25, no. 3, pp. 2017-2024, 2010.

[16] M. Korkali, H. Lev-Ari, and A. Abur, "Traveling-wave-based faultlocation technique for transmission grids via wide-area synchronized voltage measurements," IEEE Transactions on Power Systems, vol. 27, no. 2, pp. 1003-1011, 2012.

[17] C. Draeger, J. Aime, and M. Fink, "One-channel time-reversal in chaotic cavities: Experimental results," The Journal of the Acoustical Society of America, vol. 105, p. 618, 1999.

[18] A. Derode, A. Tourin, J. de Rosny, M. Tanter, S. Yon, and M. Fink, "Taking advantage of multiple scattering to communicate with timereversal antennas," Physical Review Letters, vol. 90, no. 1, p. 14301, 2003.

[19] A. Cozza, "Statistics of the performance of time reversal in a lossy reverberating medium," Physical Review E, vol. 80, no. 5, p. 056604, 2009.

[20] E. Wolf, Introduction to the Theory of Coherence and Polarization of Light. Cambridge University Press, 2007.

[21] Z. Wang, R. Razzaghi, M. Paolone, and F. Rachidi, "Electromagnetic time reversal applied to fault location: On the properties of back-injected signals," in 2018 Power Systems Computation Conference (PSCC), June 2018, pp. 1-7.

[22] H. Vallon, A. Cozza, F. Monsef, and A. S. Chauchat, "Time-reversed excitation of reverberation chambers: Improving efficiency and reliability in the generation of radiated stress," IEEE Trans. Electromagn. Compat., vol. 58, no. 2, pp. 364-370, April 2016.

[23] A. Derode, P. Roux, and M. Fink, "Robust acoustic time reversal with high-order multiple scattering," Physical Review Letters, vol. 75, no. 23 , pp. 4206-4209, 1995.

[24] A. Derode, A. Tourin, and M. Fink, "Random multiple scattering of ultrasound. II. Is time reversal a self-averaging process?" Physical Review E, vol. 64, no. 3, p. 36606, 2001. 